\title{
The complicated clinical course in a case of atypical lipodystrophy after development of neutralizing antibody to metreleptin: treatment with setmelanotide
}

\author{
Baris Akinci1,2, Rasimcan Meral1, Diana Rus', Rita Hench1, Adam H Neidert1, Frank DiPaola³, Maria Westerhoff4, \\ Simeon I Taylor 5 and Elif A Oral ${ }^{1}$ \\ 1Brehm Center for Diabetes Research and Division of Metabolism, Endocrinology \& Diabetes, University of Michigan, \\ Ann Arbor, Michigan, USA, 2Division of Endocrinology and Metabolism, Dokuz Eylul University, Izmir, Turkey, ${ }^{3}$ Division \\ of Pediatric Gastroenterology, University of Michigan, Ann Arbor, Michigan, USA, 4Department of Pathology, \\ University of Michigan, Ann Arbor, Michigan, USA, and 5Division of Endocrinology, Diabetes, and Nutrition, University \\ of Maryland School of Medicine, Baltimore, Maryland, USA
}

Correspondence should be addressed to E A Oral

Email

eliforal@med.umich.edu

\begin{abstract}
Summary
A patient with atypical partial lipodystrophy who had a transient initial response to metreleptin experienced acute worsening of her metabolic state when neutralizing antibodies against metreleptin appeared. Because her metabolic status continued to deteriorate, a therapeutic trial with melanocortin-4 receptor agonist setmelanotide, that is believed to function downstream from leptin receptor in the leptin signaling system, was undertaken in an effort to improve her metabolic status for the first time in a patient with lipodystrophy. To achieve this, a compassionate use (investigational new drug application; IND) was initiated (NCT03262610). Glucose control, body fat by dual-energy X-ray absorptiometry and MRI, and liver fat by proton density fat fraction were monitored. Daily hunger scores were assessed by patient filled questionnaires. Although there was a slight decrease in hunger scales and visceral fat, stimulating melanocortin-4 receptor by setmelanotide did not result in any other metabolic benefit such as improvement of hypertriglyceridemia or diabetes control as desired. Targeting melanocortin-4 receptor to regulate energy metabolism in this setting was not sufficient to obtain a significant metabolic benefit. However, complex features of our case make it difficult to generalize these observations to all cases of lipodystrophy. It is still possible that melanocortin-4 receptor agonistic action may offer some therapeutic benefits in leptin-deficient patients.
\end{abstract}

\section{Learning points:}

- A patient with atypical lipodystrophy with an initial benefit with metreleptin therapy developed neutralizing antibodies to metreleptin (Nab-leptin), which led to substantial worsening in metabolic control. The neutralizing activity in her serum persisted for longer than 3 years.

- Whether the worsening in her metabolic state was truly caused by the development of Nab-leptin cannot be fully ascertained, but there was a temporal relationship. The experience noted in our patient at least raises the possibility for concern for substantial metabolic worsening upon emergence and persistence of Nab-leptin. Further studies of cases where Nab-leptin is detected and better assay systems to detect and characterize Nab-leptin are needed. 
- The use of setmelanotide, a selective MC4R agonist targeting specific neurons downstream from the leptin receptor activation, was not effective in restoring metabolic control in this complex patient with presumed diminished leptin action due to Nab-leptin.

- Although stimulating the MC4R pathway was not sufficient to obtain a significant metabolic benefit in lowering triglycerides and helping with her insulin resistance as was noted with metreleptin earlier, there was a mild reduction in reported food intake and appetite.

- Complex features of our case make it difficult to generalize our observation to all leptin-deficient patients. It is possible that some leptin-deficient patients (especially those who need primarily control of food intake) may still theoretically benefit from MC4R agonistic action, and further studies in carefully selected patients may help to tease out the differential pathways of metabolic regulation by the complex network of leptin signaling system.

\section{Background}

Lipodystrophy syndromes are a heterogeneous cluster of diseases characterized by loss of subcutaneous adipose tissue that leads to severe insulin resistance and dyslipidemia. Metreleptin therapy is now an approved treatment for generalized lipodystrophy in the United States. The European Medicines Agency (EMA) has approved metreleptin for generalized lipodystrophy and also for selected patients with partial lipodystrophy. An important complication of metreleptin therapy is the potential emergence of neutralizing antibodies (Nableptin). There are only sparse data on the clinical relevance of Nab-leptin: details on how to detect and interpret assay data and whether these antibodies truly block in vivo leptin action are unclear (1).

Here, we report the complicated course of the disease and investigational treatment efforts in a patient with an atypical form of partial lipodystrophy who developed Nab-leptin when she was being treated with metreleptin in the context of a clinical study testing the efficacy of this drug for the liver disease associated with partial lipodystrophy. While the patient had initially demonstrated improvement in insulin resistance and liver histopathology with metreleptin, there was substantial worsening even beyond baseline state following the emergence of Nab-leptin.

We then report the treatment experience with setmelanotide, an investigational agent, chosen for its mechanistic relevance and lack of conventional treatment options. Even though the treatment result is negative in our hands, our observations are still noteworthy to provide a beginning point to discuss the complicated nature of signaling system for metabolic actions of leptin based on the unique opportunities of our case.

\section{Case presentation Description of the patient}

The patient is a 17-year-old Caucasian female with atypical partial lipodystrophy, type 1 diabetes, nonalcoholic steatohepatitis (NASH), and severe hypertriglyceridemia (Fig. 1A). The patient was seen for the first time at age 12 . She demonstrated partial fat loss (2), mainly affecting the limbs (Fig. 1B). Her initial lab tests are shown in Table 1. Leptin levels $(1.2 \mathrm{ng} / \mathrm{mL})$ initially measured with RIA were lower than predicted for adiposity. The University of Chicago panel was negative for known lipodystrophy genes (Table 2). Whole exome sequencing (WES) revealed several variants of unknown significance in genes of interest, but not in known lipodystrophy genes (Table 2). Metreleptin therapy was started to treat her high triglycerides and severe hepatic steatosis with informed consent as part of a National Institutes of Health (NIH) funded clinical trial NCT01679197, testing the efficacy of this drug in the liver disease associated with partial lipodystrophy. During the first 12 months, metabolic parameters showed improvement after metreleptin (Table 3), especially during the mixed-meal test (Fig. 2A), with improved postmeal glucose excursions and insulin sensitivity. More importantly, her liver biopsy demonstrated a reduction in steatosis, hepatic injury, as well as fibrosis (Fig. 2B). She continued metreleptin as part of a longer treatment protocol (NCT02654977) due to its clinical benefit.

\section{Investigation}

\section{Development of Nab-leptin and unmet medical need}

Around 18 months of therapy with metreleptin, the patient presented with acute hyperglycemia and 
hypertriglyceridemia (Fig. 1C, shown with * on the figure). Surprisingly, beta-hydroxybutyrate level was elevated. She had elevated titers of anti-GAD, and her C-peptide was $<0.01 \mathrm{ng} / \mathrm{mL}$ (reference: $1.0-5.2 \mathrm{ng} / \mathrm{mL}$ ). She was diagnosed with type 1 diabetes. The suspicion for Nableptin was raised upon noticing an undetectable leptin level which persisted $1 \mathrm{~h}$ after an administered dose of metreleptin. The presence of Nab-leptin was ultimately confirmed by the assays that are described previously (1), conducted by the manufacturer of metreleptin (Aegerion Pharmaceuticals, Cambridge, MA). The level of in vitro neutralization was reported to be Category $\mathrm{E}$, the highest possible activity. Metreleptin was discontinued as requested by the Food and Drug Administration (FDA) upon reporting of this complication. Her clinical course was further complicated with emergence of a picture suggestive of autoimmune hepatitis when metreleptin was eventually tapered and discontinued and alternative drugs were used (Fig. 2C). Subsequently, her glucose and lipid control became substantially worse, and she required multiple hospitalizations for episodes of severe hyperglycemia, diabetic ketoacidosis, and pancreatitis (Fig. 1C). During these episodes, she was treated with aggressive hydration, i.v. insulin infusions at a high rate but with a limit on food intake, and a reduction in carbohydrate substrate. The patient also developed antibodies against insulin (detected a few years after she started insulin treatment) but the titers were low (0.14 $\mathrm{nmol} / \mathrm{L}$; normal: $<0.02)$. We concluded that neither insulin antibodies nor insulin receptor antibodies were sufficiently explanatory to her clinical picture, considering the lack of severe hypoglycemic episodes and hypercatabolism and the presence of progressive hepatic steatosis.

\section{Treatment}

\section{Compassionate use protocol}

The patient's serious and life-threatening disease and the lack of satisfactory alternatives to treat her condition led us to seek additional therapies. We, therefore, sought treatment with setmelanotide in this single patient (NCT03262610) under institutional review board (IRB) and FDA oversight (Ann Arbor, MI; 8/15/2017 01/05/2018). The patient and mother gave consent to be treated. The efficacious dose in genetic obesity patients previously treated in the setmelanotide clinical trial program had ranged from total daily doses of 1.0 to 2.5 mg of setmelanotide. However, due to the small number of genetic obesity patients exposed to setmelanotide, careful increases in dose titrations were conducted in this single patient study of lipodystrophy, a disorder associated with deficiency of signaling through the leptin-melanocortin pathway but not characterized by extreme obesity. Setmelanotide (supplied by Rhythm Pharmaceuticals, Boston, MA) was started at $1 \mathrm{mg} /$ day and the dose was titrated up to a maximum dose of $3 \mathrm{mg} /$ day. Successful metabolic amelioration was defined as achieving triglyceride levels $<500 \mathrm{mg} / \mathrm{dl}$ without the need for plasmapheresis. Other predetermined clinical benefits were a reduction in $\mathrm{HbA} 1 \mathrm{c}>0.5 \%$ and a substantial reduction in insulin dose.

Efficacy measurements included lipid panel, glycemic parameters, liver fat, and visceral quantification by MRI (Philips 3T, Ingenia SW R5.3) and body composition assessed by dual-energy X-ray absorptiometry (DXA) scan (2) (GE Lunar Prodigy, model PA +41744 , Madison, WI) at baseline and the end of the treatment period. Changes from baseline in weight $(\mathrm{kg})$, waist and hip circumferences $(\mathrm{cm})$, blood pressure $(\mathrm{mmHg})$, and heart rate (bpm) were recorded. Hunger scores were assessed by Hunger Questionnaire and Global Hunger Questions (Table 4). Hunger assessments were recorded on a daily basis, prior to the patient's morning meal. Other questionnaires included the Short Form (SF)-36 Health Questionnaire, Patient Health Questionnaire-9, and Columbia-Suicide Severity Rating Scale. Clinical safety laboratory tests included complete blood count, chemistry, urinalysis, and coagulation profile, and 12-lead ECG. The patient was monitored for the development of any adverse event throughout the treatment. Comprehensive skin examinations were performed by a dermatologist.

\section{Outcome and follow-up}

The treatment with setmelanotide did not result in convincing metabolic amelioration and the patient did not reach any of the predetermined criteria. Although there was a slight decrease in hunger scales, this did not translate into any weight loss (Fig. 1D). The patient also gained weight between day 84 and 144 of therapy. Similarly, setmelanotide did not improve glycemic control and hypertriglyceridemia in a sustained manner (Table 3). A slight decrease in total daily insulin dose prompted by occurrence of hypoglycemia (glucose levels $<50 \mathrm{mg} / \mathrm{dL}$ ( $2.78 \mathrm{mmol} / \mathrm{L}$ ) on multiple occasions) was not considered clinically significant, as HbA1c levels remained high despite administration of more 2.5 units/kg of insulin/ day (Fig. 1E). Setmelanotide showed no effect on liver fat content; however, visceral fat slightly decreased (Fig. 1F). Quality-of-life scores were similar (Fig. 1G). 

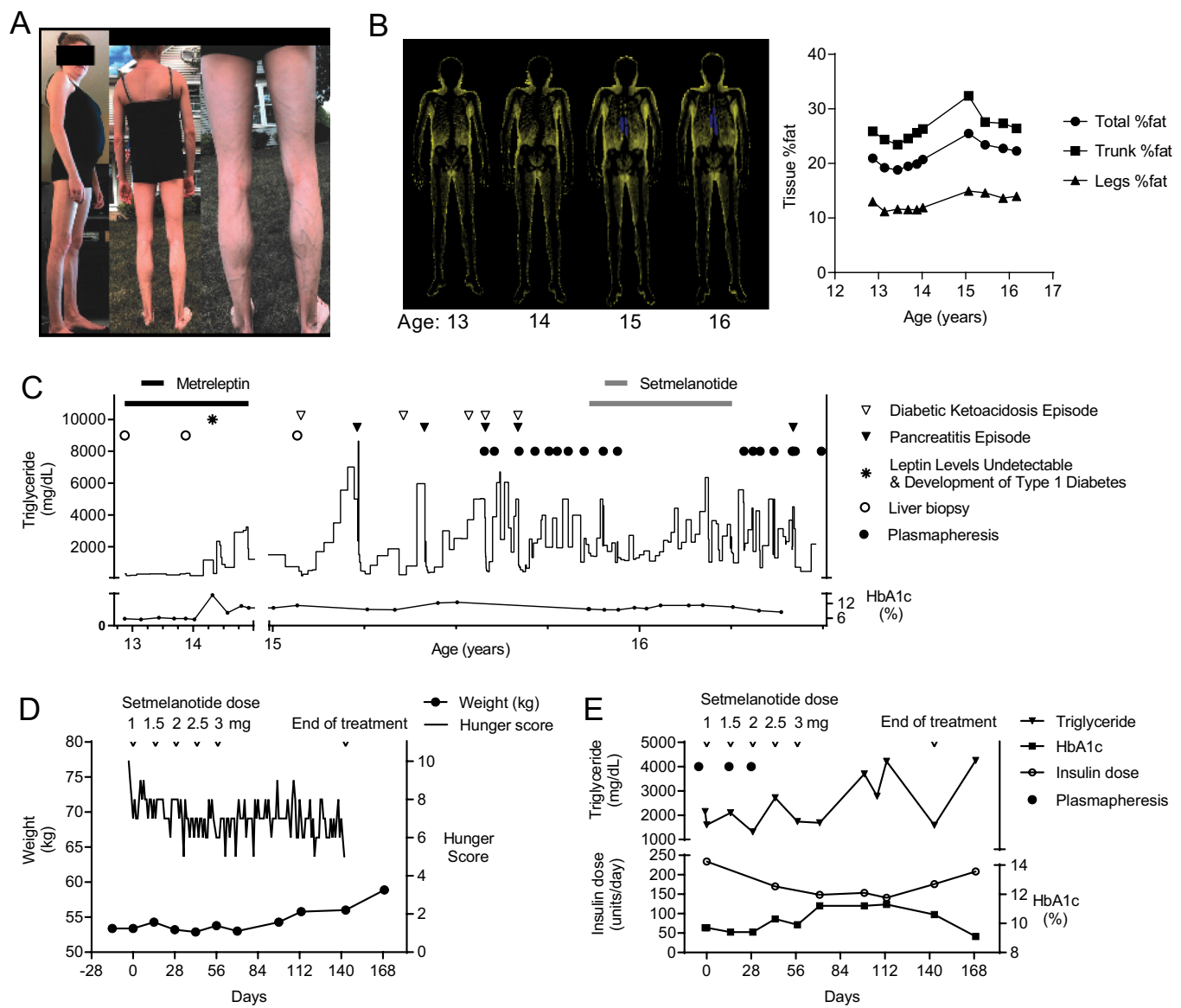

F Before setmelanotide
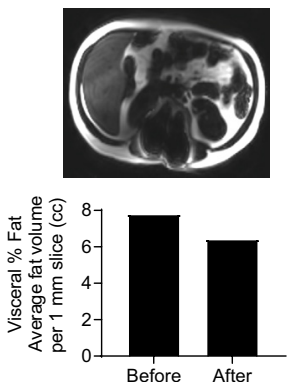

Three months after setmelanotide
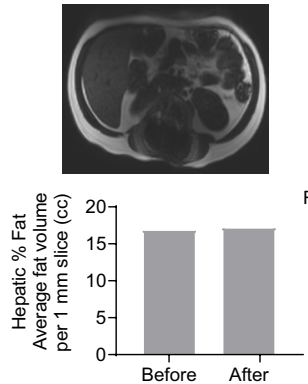

G

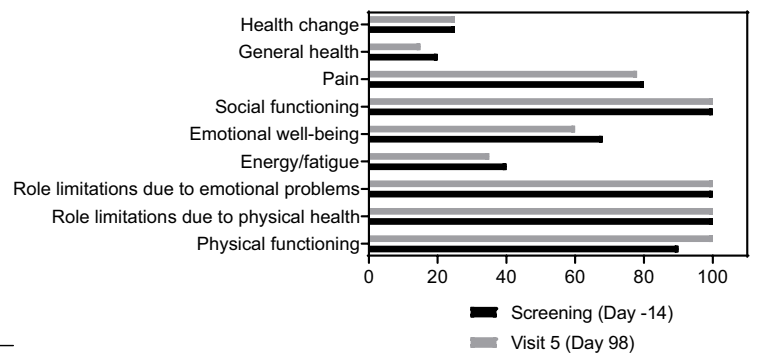

Figure 1

Patient description and compassionate treatment efforts. (A) Patient pictures showing atypical partial lipodystrophy at her presentation. Briefly, her mother began noticing a change in her body shape with thin arms and legs, and physical examination revealed lack of fat in her extremities with preservation of her trunk, face, and neck at age 11 when she was evaluated by her primary care physician for body changes that had occurred over the last year, enlarged liver and high triglycerides. She also had scoliosis and hand contractures. She had only Tanner stage 2 breast development and pubic hair, but no menstrual cycles. Her gonadal hormones indicated hypogonadotropic hypogonadism. Her morning cortisol, IGF-1, and thyroid functions were within normal limits (Table 1). Glutamic acid decarboxylase (GAD) antibodies were elevated at presentation. She demonstrated slightly low levels of Complement 4 (C4), and her Antinuclear Antibody (ANA) was positive. Family history was significant for atypical form of systemic lupus erythematosus (SLE) in her father and sudden cardiac death in his thirties. Mother had obesity and hypothyroidism. (B) Serial 'fat shadow' images obtained from dual-energy X-ray absorptiometry (DXA) scan showing partial lipodystrophy (2). Total fat percentage was reported as $21 \%$, and fat mass ratio (FMR) was 1.99 at presentation. Note the increase in total fat over time, which is due to an increase in truncal fat. Patient underwent corrective surgery for scoliosis between age 14 and 15 . It was hard to classify her lipodystrophy using the current classification schema. Although our patient's fat distribution resembles familial partial lipodystrophy, we opted to classify her as atypical partial lipodystrophy considering the age of onset, a wide range of symptoms in addition to lipodystrophy including hand contractures, scoliosis, and amenorrhea, underlying autoimmunity, and disease progression. Even though our patient was classified as atypical partial lipodystrophy, the cluster of type 1 diabetes and autoimmune hepatitis in association with an acquired form of generalized lipodystrophy is well known, which is associated with low C4 levels. Our patient has substantially more upper body adiposity and subcutaneous abdominal fat compared to those other patients previously described. Therefore, she does not quite fit any of the established patterns in the current classification system of lipodystrophy. (C) The 
Table 1 Laboratory results at initial evaluation.

\begin{tabular}{|c|}
\hline Parameter \\
\hline $\mathrm{HbA1c}(\% ; \mathrm{mmol} / \mathrm{mol})$ \\
\hline Glucose (mg/dL; mmol/L) \\
\hline Insulin (uU/mL; pmol/L) \\
\hline Triglyceride (mg/dL; mmol/L) \\
\hline Total cholesterol (mg/dL; mmol/L) \\
\hline HDL cholesterol (mg/dL; mmol/L) \\
\hline LDL cholesterol (mg/dL; mmol/L) \\
\hline Chol/HDL Ratio \\
\hline Apo-lipoprotein A-1 (mg/dL; mmol/L) \\
\hline Apo-lipoprotein B (mg/dL; mmol/L) \\
\hline Creatinine $(\mathrm{mg} / \mathrm{dL} ; \mathrm{mmol} / \mathrm{L})$ \\
\hline AST (IU/L) \\
\hline$A L T(I U / L)$ \\
\hline ALK (IU/L) \\
\hline GGTP (IU/L) \\
\hline CK (IU/L) \\
\hline Leptin $(\mathrm{ng} / \mathrm{mL})^{*}$ \\
\hline IGF-1 (ng/mL) \\
\hline Cortisol ( $\mu \mathrm{g} / \mathrm{dL})$ \\
\hline $\mathrm{FSH}(\mathrm{mlU} / \mathrm{mL})$ \\
\hline $\mathrm{LH}(\mathrm{mlU} / \mathrm{mL})$ \\
\hline Estradiol $(\mathrm{pg} / \mathrm{mL})$ \\
\hline Testosterone (ng/mL) \\
\hline FT3 $(\mathrm{pg} / \mathrm{mL})$ \\
\hline FT4 (ng/dL) \\
\hline TSH (mIU/L) \\
\hline C3 Complement (mg/dL) \\
\hline C4 Complement (mg/dL) \\
\hline Antinuclear Antibody \\
\hline GAD Antibody (units/mL) \\
\hline
\end{tabular}

\begin{tabular}{c}
\hline Value \\
\hline $5.8(40)$ \\
$99(5.5)$ \\
$225.4(1565.4)$ \\
$305(3.45)$ \\
$169(4.37)$ \\
$29(0.75)$ \\
$79(2.04)$ \\
5.8 \\
$101(0.036)$ \\
$109(0.002)$ \\
$0.4(0.035)$ \\
53 \\
73 \\
348 \\
41 \\
113 \\
1.2 \\
220 \\
9.4 \\
3.7 \\
0.4 \\
13 \\
0.06 \\
2.6 \\
1.04 \\
0.57 \\
111 \\
15.1 \\
$1 / 320($ speckled $)$ \\
$>250$ \\
\end{tabular}

\begin{tabular}{c}
\hline Reference values \\
\hline $4.2-5.8(22-40)$ \\
$70-100(3.9-5.6)$ \\
$1.0-21.0(6.9-145.8)$ \\
$<130(1.47)$ \\
$<170(4.39)$ \\
$<40(1.03)$ \\
$<90(2.33)$ \\
$<4.5$ \\
$>110(0.039)$ \\
$<105(0.002)$ \\
$0.4-0.9(0.035-0.079)$ \\
$5-60$ \\
$<35$ \\
$90-420$ \\
$8-35$ \\
$26-180$ \\
NA \\
$143-693$ \\
A.M.: $7.0-22.0$ \\
$2.0-12.0$ \\
$2-15$ \\
Prepubertal: $<20$ \\
$0.10-0.70$ \\
$1.6-3.9$ \\
$0.76-1.70$ \\
$0.3-5.5$ \\
$79-152$ \\
$16-38$ \\
Negative \\
$0-5$ \\
\end{tabular}

${ }^{\dagger}$ Reference values for serum leptin in healthy non-obese children and adolescents were previously reported by Lausten-Thomsen et al. (11); however, reference interval for ages 0-17 are not established in the relevant population. Adult females $(B M I=22): 3.3-18.3 \mathrm{ng} / \mathrm{mL}$. *Leptin level measured via RIA. ALP, alkaline phosphatase; ALT, alanine aminotransferase; AST, aspartate aminotransferase; CK, creatine kinase; FSH, follicle stimulating hormone; FT3, free triiodothyronine; FT4, free thyroxine; GAD, glutamic acid decarboxylase; GGTP, gamma-glutamyl transpeptidase; HDL, high density lipoprotein; IGF, insulin-like growth factor; LDL, low density lipoprotein; LH, luteinizing hormone; NA, not applicable; TSH, thyroid stimulating hormone.

\section{Figure 1 (Continued)}

figure shows the complicated course of the disease and investigational treatment efforts. Metreleptin was initially successful to improve nonalcoholic steatohepatitis (NASH) and to keep the patient's metabolic status stable; however, it was complicated by the development of neutralizing antibodies (Nab-leptin). At month 18, the patient reported fatigue, increased thirst, blurred vision, and polydipsia, and had lost $5 \mathrm{lbs}$ in a month. Lab results showed significant hyperglycemia and hyperlipidemia with a positive anion gap and positive ketones. The anti-GAD65 level was 5.39 nmol/L (repeat Anti-GAD65: 8.38 $\mathrm{nmol} / \mathrm{L}, 2$ months later), and leptin level, drawn 1-h post injection, was undetectable. The presence of Nab-leptin was confirmed by the assay performed by the manufacturer of metreleptin. For her type 1 diabetes, she was started on insulin at high doses plus metformin. Upon tapering and discontinuation of metreleptin, she was treated with fenofibrate for hypertriglyceridemia and pioglitazone for improving severe insulin resistance. Her liver function test results rose to a ten-fold above normal 1 month following the initiation of pioglitazone. Liver biopsy showed patchy portal and periportal inflammation that included plasma cells and interface injury, consistent with autoimmune hepatitis. She responded well to a short course of prednisone with normalization of her liver tests, but her metabolic control worsened. She was started on frequent plasmapheresis as shown, but this did not prevent recurrent episodes of pancreatitis. (D) The effect of setmelanotide on weight and hunger scores. Note that patient had mild reduction in hunger scores while on setmelanotide. Daily Hunger Questionnaire scores were assessed by asking the patient to score her hunger based on a Likert-like scale, where 0 is not hungry at all and 10 is the hungriest possible. These were completed on 3 separate days during screening and everyday thereafter during the setmelanotide treatment period prior to dosing in the morning (fasted). Hunger questionnaire and scales are presented in Table 4. Data shown are from scores on Question 2 (analyses of other questions showed a similar profile with no additional data shown). Also, no change was observed in the Global Hunger Assessment (data not shown). (E) The effect of setmelanotide on triglycerides, $\mathrm{HbA} 1 \mathrm{c}$, and total daily insulin. Because the patient developed hypoglycemia upon initiation of setmelanotide, her insulin dose was reduced to prevent further hypoglycemia. The triglyceride levels were somewhat lower in the earlier part of the therapy, but the effect was not sustained. By the end of pre-specified treatment period, it was necessary to re-initiate plasmapheresis. (F) The effect of setmelanotide on the liver and visceral fat content. Axial magnetic resonance (MR) images are presented as well as quantification. Liver fat reported as proton-density fat fraction and visceral fat were quantified as described previously. There was no real change in liver fat and a slight reduction in visceral fat using this short-term treatment. (G) The effect of setmelanotide on quality-of-life parameters. The summary assessments of Short Form (SF)-36 version 1 (v1) Health Questionnaire at baseline and after setmelanotide therapy are presented, showing no real change with therapy. 
Table 2 The list of known lipodystrophy genes included in the University of Chicago panel.

\begin{tabular}{l} 
The University of Chicago Panel \\
\hline AGPAT2 \\
AKT2 \\
BSCL2 \\
CAV1 \\
CIDEC \\
LMNA \\
PLIN1 \\
PPARG \\
PTRF \\
TBC1D4 \\
ZMPSTE24
\end{tabular}

No adverse events directly attributable to setmelanotide were observed except skin discoloration (tanning) and mild hypoglycemia, leading to insulin dose titrations. No increases in heart rate or blood pressure at any dose were noted. Although the reported systolic blood pressure is higher before setmelanotide (Table 3 ), we should note that it only reflects the values of 1-day measurements which could be affected by several transient factors. Subsequent measurements of blood pressure were within the normal range and no clinically significant fluctuations were seen. Also, no adverse signal was observed in psychological health assessments.
Of note, Category E Nab-leptin persisted throughout the time-course covered in this manuscript after its first detection. We did not note preponderance to recurrent severe infections in this patient, despite the colonization with group B streptococci in the urinary tract and development of a pseudocyst in the abdomen after recurrent pancreatitis episodes during the pretreatment period.

\section{Discussion}

Although the development of Nab-leptin has been previously identified in patients with lipodystrophy treated with metreleptin (1), there have been no long-term reports of the metabolic or other clinical states of patients who presented with Nab-leptin. Here we first describe the long-term metabolic complications that have emerged after development of Nab-leptin, despite demonstration of initial benefit with metreleptin therapy. We then report the treatment experience with setmelanotide, an investigational agent, chosen for its mechanistic relevance and lack of conventional treatment options. Setmelanotide is a melanocortin-4 receptor (MC4R) agonist that retains the specificity and functionality of the naturally occurring Pro-opiomelanocortin (POMC)-

Table 3 Clinical and laboratory characteristics of the patient before and after metreleptin and setmelanotide therapies.

\begin{tabular}{l}
\hline \\
\hline Age \\
Height (m) \\
Weight (kg) \\
BMI (kg/m²) \\
Waist (cm) \\
Blood Pressure (Sys:Dia mmHg) \\
Pulse (per min) \\
$\%$ Body Fat \\
FMR \\
$\%$ Liver Fat (PDFF) \\
Triglyceride (mg/dL; mmol/L) \\
Cholesterol (mg/dL; mmol/L) \\
HDL (mg/dL; mmol/L) \\
LDL (mg/dL; mmol/L) \\
Hemoglobin A1c (\%; mmol/mol) \\
Daily total insulin dose (units) \\
Creatinine (mg/dL, mmol/L) \\
ALT (IU/L) \\
AST (IU/L) \\
ALP (IU/L) \\
Leptin (ng/mL)*
\end{tabular}

\begin{tabular}{c}
\hline Before metreleptin \\
\hline 12 years and \\
10 months \\
1.54 \\
41.3 \\
17.4 \\
74 \\
$117: 67$ \\
86 \\
21.00 \\
1.99 \\
5.00 \\
$305(3.45)$ \\
$169(4.37)$ \\
$29(0.75)$ \\
$79(2.04)$ \\
$5.8(40)$ \\
None \\
$0.44(0.039)$ \\
73 \\
53 \\
348 \\
7.4
\end{tabular}

\begin{tabular}{c}
\hline year after metreleptin \\
\hline 13 years and \\
10 months \\
1.60 \\
46.8 \\
18.3 \\
77 \\
$121: 62$ \\
71 \\
19.90 \\
2.23 \\
3.48 \\
$303(3.42)$ \\
$142(3.67)$ \\
$28(0.72)$ \\
$53(1.37)$ \\
$5.9(41)$ \\
None \\
$0.54(0.048)$ \\
31 \\
31 \\
293 \\
271.7
\end{tabular}

\begin{tabular}{c}
\hline Before setmelanotide \\
\hline 15 years and \\
10 months \\
165 \\
53.4 \\
19.6 \\
85.2 \\
$136: 82$ \\
82 \\
22.74 \\
2.00 \\
16.3 \\
$1601(18.09)$ \\
$239(6.18)$ \\
$25(0.65)$ \\
$28(0.72)$ \\
$9.7(83)$ \\
234 \\
$0.56(0.049)$ \\
34 \\
44 \\
116 \\
$<0.6$
\end{tabular}

\begin{tabular}{c} 
End of setmelanotide \\
\hline 16 years and \\
2 months \\
166 \\
56 \\
20.4 \\
83.6 \\
$111: 78$ \\
87 \\
22.29 \\
1.89 \\
19.9 \\
$1590(17.97)$ \\
$199(5.15)$ \\
$24(0.62)$ \\
$32(0 / 83)$ \\
$10.6(92)$ \\
176 \\
$0.57(0.050)$ \\
50 \\
41 \\
136 \\
$<0.6$
\end{tabular}

*Leptin level measured via ELISA

ALP, alkaline phosphatase; ALT, alanine aminotransferase; AST, aspartate aminotransferase; FMR, fat mass ratio; GGTP, gamma-glutamyl transpeptidase; HDL, high density lipoprotein; LDL, low density lipoprotein; NA, not applicable; PDFF, proton density fat fraction. 
A
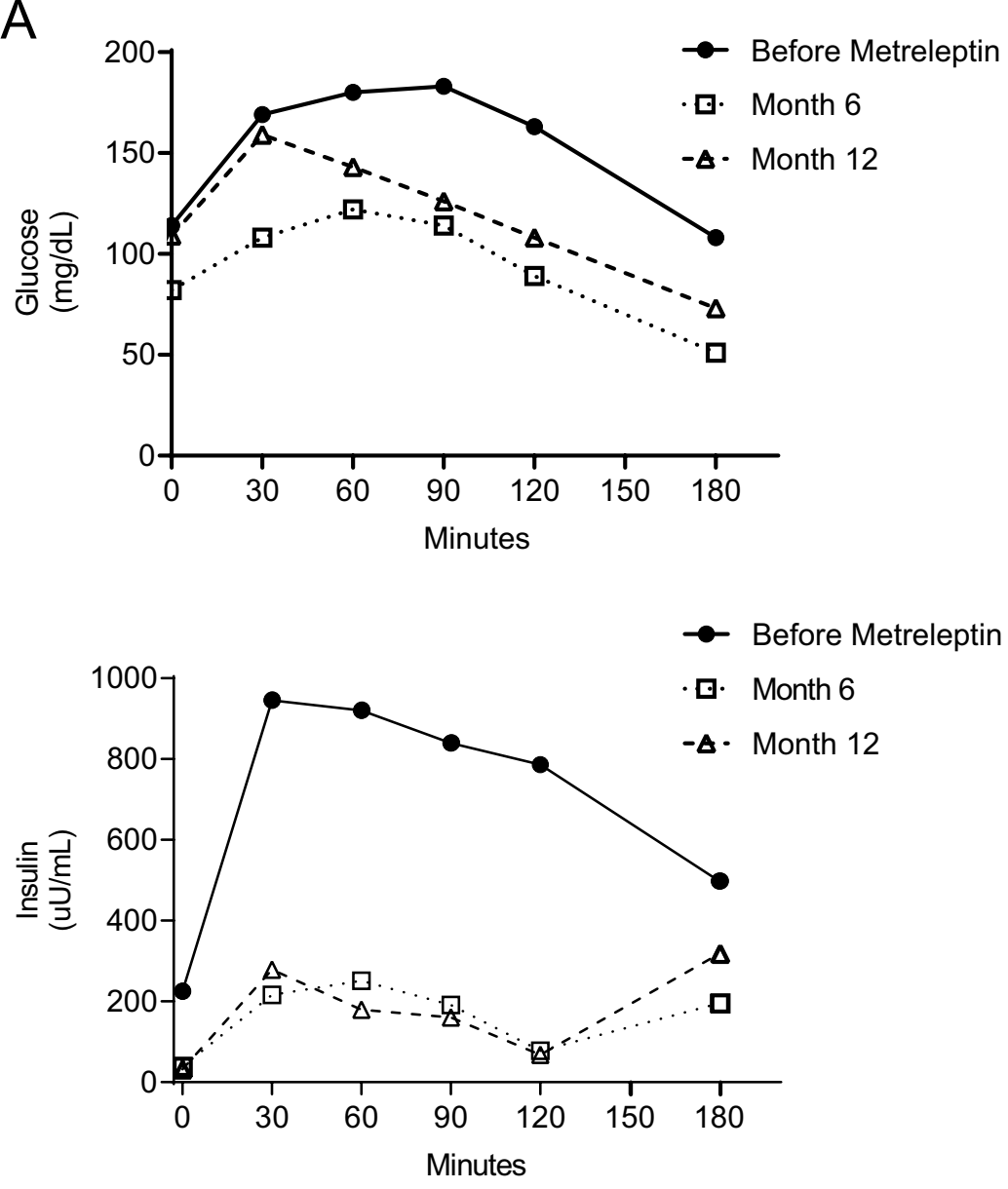

B Baseline

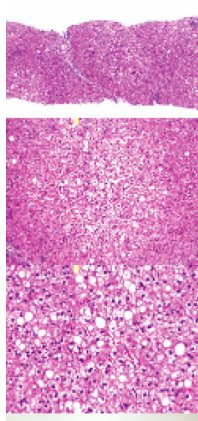

12 month
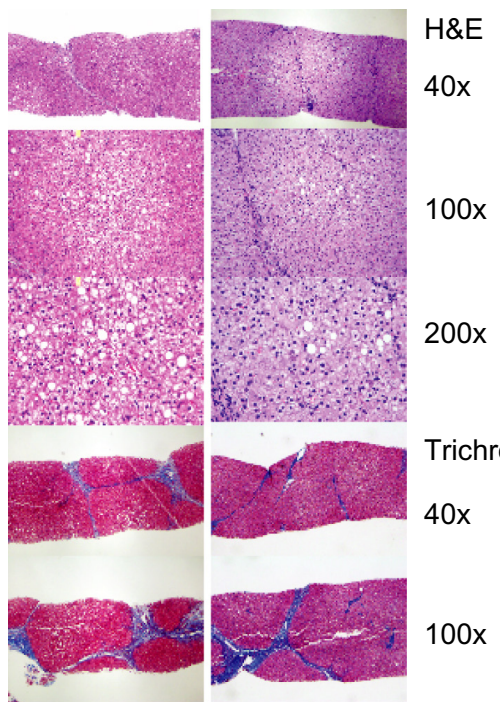

Trichrome

$40 x$

$100 x$

C

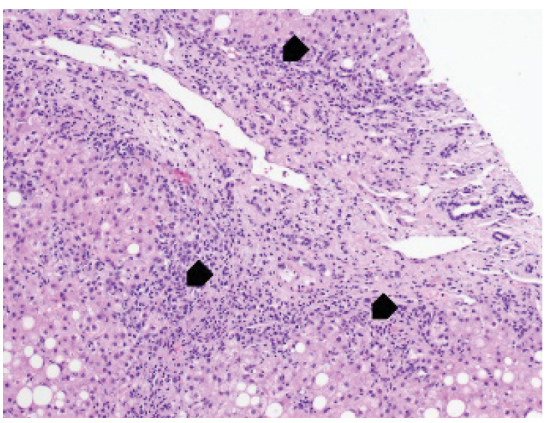

Figure 2

(A) Mix meal test before and after metreleptin. (B) Liver biopsy before and 12 months after metreleptin. The liver biopsy prior to metreleptin shows steatohepatitis with bridging fibrosis and cirrhosis by histology in the area that was biopsied. NASH scoring before metreleptin: Fibrosis 4, hepatocellular injury 2, steatosis 2, parenchymal inflammation 1, and total NASH score: 9 and after metreleptin: fibrosis 3, injury 2, steatosis 1, inflammation 1, and total NASH score 7. (C) Liver biopsy showing evidence for autoimmune hepatitis obtained when liver tests results were elevated ten-fold. This acute elevation in liver tests occurred upon discontinuation of metreleptin and sequential addition of fenofibrate and pioglitazone. Both agents were then discontinued and patient was treated with a short course of prednisone. Arrow heads indicate bile ductular reaction with mild chronic inflammation, including plasma cells.

derived neuropeptide, $\alpha$-MSH, which is the natural ligand for MC4R (3). In previous studies, body weight and hunger scores were reduced in POMC and leptin receptor (LEPR) deficient obesity patients treated with setmelanotide (4, 5). Adverse events in these patient populations included increased darkening of the skin/nevi and injection site reactions without long-term consequences.

The MC4R signaling system is an important mediator of leptin's action in the CNS $(6,7)$. The balance between different types of leptin-sensitive neurons that are scattered across the brain collectively regulate the energy intake and expenditure and also control other pleiotropic actions of leptin. Leptin activates POMC neurons and increases levels of $\alpha$-MSH (6). Leptin's glucose normalizing effect in type 1 diabetes was also dependent upon activation of
POMC neurons in rodent models (8), even though leptin did not play a substantial role in improving glucose in human type 1 diabetes in the only trial reported to date (9). In this compassionate use protocol, the proposed clinical benefits of setmelanotide were predetermined as important endpoints for this patient and were previously observed to be affected by leptin in lipodystrophy. The hypothesis that we wanted to test specifically was whether those known clinical effects of leptin in lipodystrophy were being mediated by the MC4R system in this patient. However, in our hands, stimulating MCR4 system by setmelanotide did not work as desired. Taken all data together, it is possible to conclude either that the MC4R pathway did not directly mediate the initial improvements of insulin sensitivity and liver histopathology observed in 
Table 4 Hunger questionnaires.

Daily Hunger Questionnaire: This questionnaire asks you about your current hunger, as well as your hunger over the past day (last 24 h). Please answer each question every morning, around the same time of day, prior to dosing. Please answer as accurately as possible.

Please circle a number for each question:

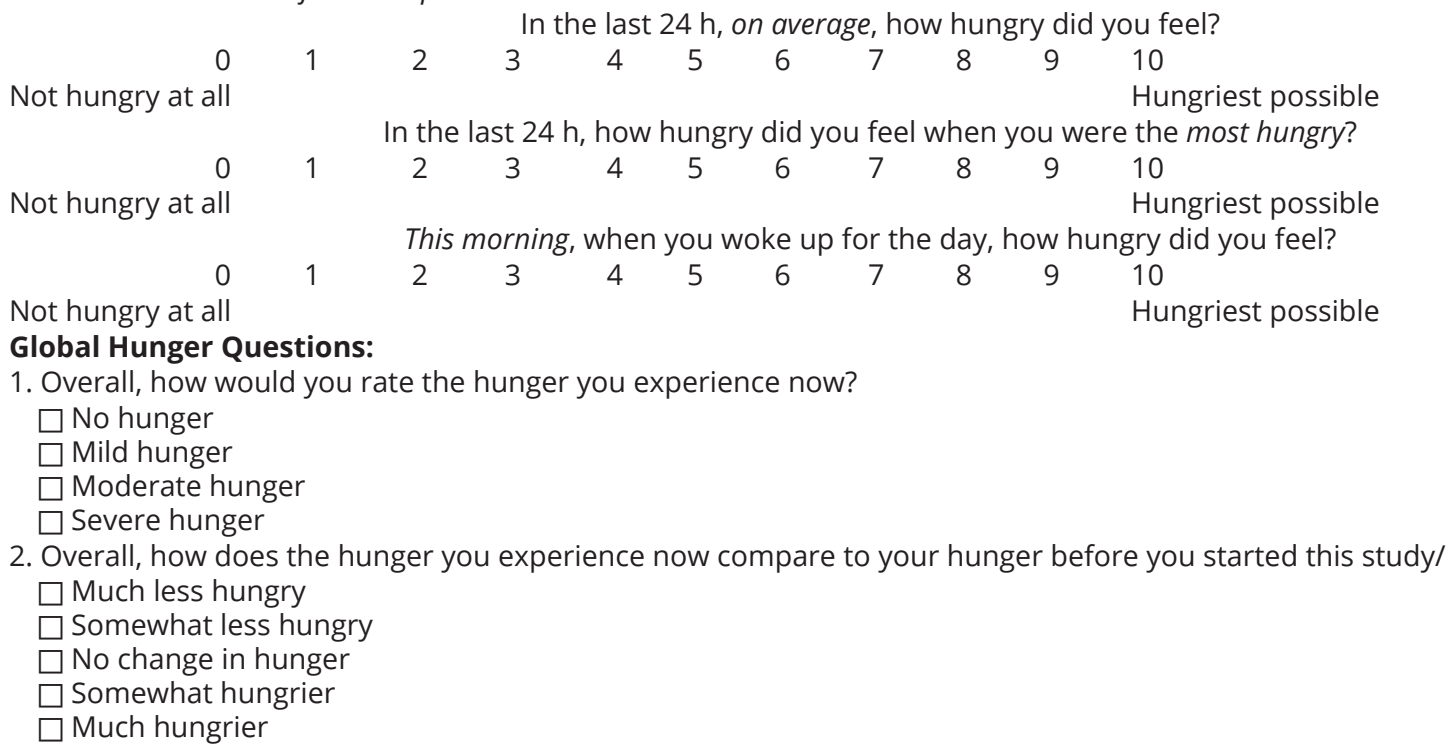

our patient or that our patient had some unique factors potentially negatively regulating this pathway (such as insulin deficiency or other unknown factors). While MC4R is one of the pathways of leptin action, our observations suggest that leptin's actions on these pathways may be through other downstream effectors of leptin. There are numerous different leptin-receptor positive neurons in the hypothalamus as well as in other areas in the brain and it will be hard to hypothesize which specific group of neurons mediated the effects we noted in our patient. Although much of the rodent data previously suggested that the metabolic effects of leptin are mediated by the central activation of the leptin receptors in lipodystrophy models, whether there are direct peripheral effects on the immune cells or other peripheral sites such as the liver in humans with lipodystrophy remains unknown.

Our case had a number of additional metabolic lessons that are worth highlighting. First of all, it was difficult to characterize her phenotype using the current consensus classification system of lipodystrophy. She clearly had partial fat loss and not generalized absence of fat, but also demonstrated the cluster of clinical and laboratory abnormalities seen in some patients with acquired generalized lipodystrophy (10). It is possible that she has a condition more similar to acquired generalized lipodystrophy caused by complex immune dysregulation (10), which may also drive development of Nab-leptin.
Secondly, we should emphasize that her baseline relative leptin deficiency probably made it more likely that she would respond to metreleptin therapy. Thirdly, the emergence of clinical type 1 diabetes and Nab-leptin correlated temporally, but we do not have any other functional evidence for a causal association. Our patient had the highest measurable level of neutralizing activity in the Nab-leptin functional assay and whether these antibodies mediated any aspect of autoimmune activity against the beta-cells or modified her immune system in any subtle way remain unknown. Functional studies with the patient's sera in rodent models prone to development of type 1 diabetes or systems that interrogate immune activation may potentially provide some needed clues in this regard.

In conclusion, our patient provides the first long-term dataset in understanding the potential consequences of Nab-leptin. Moreover, for our complex patient, targeting the central regulation of energy homeostasis with the use of a selective MC4R agonist was not effective. We conclude that just targeting this pathway with setmelanotide is not sufficient to restore the initial improvements in insulin resistance and hepatic steatosis noted in this patient with metreleptin. However, it may be difficult to generalize our observations in this complex case with lipodystrophy to all patients with diminished leptin action. Potentially, a certain group of hypoleptinemic patients may still benefit 
from MC4R agonistic action. Also, further studies are underway to understand if activating the leptin receptor with an agonist will be more effective.

\section{Declaration of interest}

B A has attended Scientific Advisory Board Meetings organized by Aegerion Pharmaceuticals and has received honoraria as a speaker from AstraZeneca, Lilly, MSD, Novartis, Novo Nordisk, Boehringer-Ingelheim, Servier, and Sanofi-Aventis. E A O reports the following conflicts: Grant support: Aegerion Pharmaceuticals, Ionis Pharmaceuticals, Akcea Therapeutics, Gemphire Therapeutics, and GI Dynamics (current) and AstraZeneca (past 2 years). Consultant or Advisor: AstraZeneca, Thera Therapeutics, and BMS (past) and Regeneron and Aegerion (current). Drug support: Aegerion Pharmaceuticals, Akcea Therapeutics, and Rhythm Pharmaceuticals (all current). Other support: Aegerion Pharmaceuticals (current). S I T has received payments for consulting services from Ionis Pharmaceuticals. The other authors have nothing to disclose.

\section{Funding}

The investigations obtained during metreleptin treatment in this patient were supported by National Institutes of Health (NIH) grant R01 DK088114. Metreleptin was provided by Amylin Pharmaceuticals and is now owned by Aegerion Pharmaceuticals. Setmelanotide and funding for the MRI imaging during setmelanotide therapy were provided in kind by Rhythm Pharmaceuticals. Other infrastructure and data management support have been provided by the NIH Clinical and Translational Science Awards grant UL1TR000433, the Nutrition Obesity Research Centers grant P30 DK089503, and NIH institutional grant DK034933. R M was supported by the University of Michigan Lipodystrophy Fund gifted by the Sopha Family and the White Point Foundation of Turkey.

\section{Patient consent}

We have obtained informed consent from the patient and the patient's guardian for the publication of this article and the accompanying images.

\section{Acknowledgements}

The authors thank the patient and her family for the willingness to receive investigational medications for her condition. We are grateful to Fred Fiedorek, MD, and Matthew Webster from Rhythm Pharmaceuticals who provided support for drug and the establishment of the compassionate use IND. The authors also appreciate Misty Gravelin and Kevin Weatherwax from the MIAP team of MICHR for their assistance in the regulatory aspects of this compassionate use protocol. The entire staff at Michigan Clinical Research Unit went above and beyond the call of duty to assist in the treatment of this patient. Also, the Pediatric Intensive Care Unit of Bronson Hospital in Kalamazoo provides for their exceptional care for this patient.

\section{References}

1 Chan JL, Koda J, Heilig JS, Cochran EK, Gorden P, Oral EA \& Brown RJ. Immunogenicity associated with Metreleptin treatment in patients with obesity or lipodystrophy. Clinical Endocrinology $2016 \mathbf{8 5}$ 137-149. (https://doi.org/10.1111/cen.12980)

2 Meral R, Ryan BJ, Malandrino N, Jalal A, Neidert AH, Muniyappa R, Akinci B, Horowitz JF, Brown RJ \& Oral EA. 'Fat Shadows' from DXA for the qualitative assessment of lipodystrophy: when a picture is worth a thousand numbers. Diabetes Care $2018412255-2258$. (https://doi.org/10.2337/dc18-0978)

3 Gautron L, Elmquist JK \& Williams KW. Neural control of energy balance: translating circuits to therapies. Cell 2015161 133-145. (https://doi.org/10.1016/j.cell.2015.02.023)

4 Kuhnen P, Clement K, Wiegand S, Blankenstein O, Gottesdiener K, Martini LL, Mai K, Blume-Peytavi U, Gruters A \& Krude H. Proopiomelanocortin deficiency treated with a Melanocortin- 4 receptor agonist. New England Journal of Medicine 2016375 240-246. (https://doi.org/10.1056/NEJMoa1512693)

5 Clement K, Biebermann H, Farooqi IS, Van der Ploeg L, Wolters B, Poitou C, Puder L, Fiedorek F, Gottesdiener K, Kleinau G, et al. MC4R agonism promotes durable weight loss in patients with leptin receptor deficiency. Nature Medicine 201824 551-555. (https://doi. org/10.1038/s41591-018-0015-9)

6 Myers Jr MG \& Olson DP. Central nervous system control of metabolism. Nature 2012491 357-363. (https://doi.org/10.1038/ nature11705)

7 Farooqi IS \& O'Rahilly S. Mutations in ligands and receptors of the leptin-melanocortin pathway that lead to obesity. Nature Clinical Practice: Endocrinology and Metabolism 20084 569-577. (https://doi. org/10.1038/ncpendmet0966)

8 Fujikawa T, Berglund ED, Patel VR, Ramadori G, Vianna CR, Vong L, Thorel F, Chera S, Herrera PL, Lowell BB, et al. Leptin engages a hypothalamic neurocircuitry to permit survival in the absence of insulin. Cell Metabolism 201318 431-444. (https://doi.org/10.1016/j. cmet.2013.08.004)

9 Vasandani C, Clark GO, Adams-Huet B, Quittner C \& Garg A. Efficacy and safety of Metreleptin therapy in patients with type 1 diabetes: a pilot study. Diabetes Care 201740 694-697. (https://doi.org/10.2337/ dc16-1553)

10 Savage DB, Semple RK, Clatworthy MR, Lyons PA, Morgan BP, Cochran EK, Gorden P, Raymond-Barker P, Murgatroyd PR, Adams C, et al. Complement abnormalities in acquired lipodystrophy revisited. Journal of Clinical Endocrinology and Metabolism 200994 10-16. (https://doi.org/10.1210/jc.2008-1703)

11 Lausten-Thomsen U, Lund MAV, Frithioff-Bøjsøe C, Hedley PL, Pedersen O, Hansen T, Christiansen M \& Holm JC. Reference values for leptin/adiponectin ratio in healthy children and adolescents. Clinica Chimica Acta 2019493 123-128. (https://doi.org/10.1016/j. cca.2019.03.004)

Received in final form 11 February 2020

Accepted 5 March 2020 